\title{
Accesibilidad peatonal a los servicios educativos de la ciudad de Ibagué-Tolima, Colombia
}

\author{
Mailen Stephany Alvarez Rojas* \\ Elia Quirós Rosado* \\ José Antonio Gutiérrez Gallego*
}

Recibido el 20 de agosto de 2018; aceptado el 30 de diciembre de 2018

\begin{abstract}
This article shows the analysis of pedestrian accessibility of the school-age population between 5 and 14 years old, towards the educational centers that offer the basic primary and basic secondary levels in the city of Ibagué. For the development of the study, information was provided on the city's road network, the population census for 2005 blocks and the educational centers of the urban area with their offer for 2017. The spatial and demographic understanding of the city together with the application of minimum time indicators, absolute accessibility and cumulative opportunities, they allow for an integral analysis taking into account the supply-demand for education. Based on the results obtained, a diagnosis is generated that evaluates the degree of accessibility that the school-age population has and the coverage that the educational centers have over the territory.
\end{abstract}

Key words: Pedestrian accessibility, basic education, geographic information systems, accessibility indicators.

\section{Resumen}

Este artículo muestra el análisis de accesibilidad peatonal de la población en edad escolar entre los 5 y 14 años, hacia los centros educativos que ofrecen los niveles de básica primaria y básica secundaria en la ciudad de Ibagué. Para el desarrollo del estudio, se contó con información de la red vial de la ciudad, el censo poblacional

* Universidad de Extremadura, Departamento de Expresión Gráfica, Escuela Politécnica, Avda. de la Universidad s/n 10071 Cáceres, España, correo electrónico:

malvarezpv@alumnos.unex.es 
por manzanas de 2005 y los centros educativos del casco urbano con su oferta hacia el 2017. La comprensión espacial y demográfica de la ciudad junto con la aplicación de los indicadores de tiempos mínimos, accesibilidad absoluta y oportunidades acumuladas, permiten realizar un análisis integral teniendo en cuenta la oferta -demanda educativa. A partir de los resultados obtenidos, se genera un diagnóstico que evalúa el grado de accesibilidad que posee la población en edad escolar y la cobertura que los centros educativos tienen sobre el territorio.

Palabras clave: Accesibilidad peatonal, educación básica, sistemas de información geográfica, indicadores de accesibilidad.

\section{Resumo}

Este artigo mostra a análise da acessibilidade pedonal da população em idade escolar entre 5 e 14 anos, em direção aos centros educacionais que oferecem os níveis básico primário e fundamental básico da cidade de Ibagué. Para o desenvolvimento do estudo, foram fornecidas informações sobre a malha viária da cidade, o censo demográfico para maçãs em 2005 e os centros educacionais do centro urbano com oferta para 2017. A compreensão espacial e demográfica da cidade juntamente com a aplicação de indicadores de tempo mínimo, acessibilidade absoluta e oportunidades acumuladas, permitem uma análise integral levando em conta a oferta-demanda por educação. Com base nos resultados obtidos, gera-se um diagnóstico que avalia o grau de acessibilidade da população em idade escolar e a cobertura que os centros educacionais têm sobre o território.

Palavras-chave: Acessibilidade pedestre, educação básica, sistemas de informação geográfica, indicadores de acessibilidade.

\section{Introducción}

Para Colombia, la educación es la formación permanente de cada individuo por medio de la concepción integral de los aspectos culturales, sociales, legales y humanos. Dicha formación inicia con la educación básica la cual atiende a la primera infancia y la adolescencia dentro de la clasificación del sistema educativo nacional (Ministerio de Educación Nacional, 2010). En este estudio, se muestra el diagnóstico a 2017 de la educación desde el nivel internacional hasta el nivel local (United Nations Educational, Scientific and Cultural Organization, UNESCO, 2011).

El factor desarrollado en pro de la equidad social y educativa en este artículo es la accesibilidad geográfica, evaluando las oportunidades que la población posee para acceder al servicio educativo. Para generar un producto claro sobre esta investigación, se analiza la influencia de la accesibilidad sobre dicho servicio en Ibagué, por medio de estudios previos realizados a nivel nacional e internacional, tanto para este 
como para otros (salud, comercio, industria). Adicionalmente, se aplica un análisis de accesibilidad por medio de indicadores que se adaptan a un Sistema de Información Geográfica (SIG).

Este trabajo de investigación, muestra el análisis de accesibilidad peatonal de la población en edad escolar entre los 5 y 14 años, hacia los centros educativos que ofrecen la enseñanza en los niveles de básica primaria y básica secundaria en la ciudad de Ibagué. Para el desarrollo del estudio, se contó con la información de la red vial de la ciudad, el único censo poblacional que hasta el momento detalla su contenido por manzanas y data del año 2005 (Departamento Nacional de Estadística, 2017) y los centros educativos con su respectiva oferta hacia el 2017.

\section{Objetivos}

El principal objetivo de este estudio, es analizar la accesibilidad peatonal de la población en edad escolar a los centros de educación básica obligatoria en la ciudad de Ibagué. Para conseguirlo, la localización de dichos centros se incluye en un Sistema de Información Geográfica (SIG) que junto con la cartografía y demografía de la ciudad permiten realizar el análisis de accesibilidad a partir del enfoque espacial y cuantitativo. Así mismo, se consideran las características de oferta y demanda de cada centro y a partir de algunos indicadores de accesibilidad se evalúa la cobertura espacial de los mismos sobre la zona urbana de la ciudad.

\section{Antecedentes}

El uso de los sistemas de información geográfica durante los últimos años, ha enfocado sus esfuerzos en evaluar la accesibilidad geográfica hacía de diferentes servicios que tienen el potencial de contribuir en el mejoramiento de la calidad de vida de la población (Villanueba, 2010). Los estudios de accesibilidad espacial a partir de los SIG tienen múltiples áreas de investigación. En España por ejemplo, se han realizado estudios en el campo del transporte y el acceso a servicios desde nivel local hasta nivel nacional que permiten la implantación de nuevas formas sostenibles de desplazamiento y limita el uso del vehículo privado (Mora Aliseda, Nogales Galán, Gutierréz Gallego, y Cortés Ruíz, 2003), (Gallego, Labrador, Cabanillas, y Pintor, 2013) y (Gutiérrez Gallego, Berrocal Nieto y Ruiz Labrador, 2014).

Por otra parte, en México y Argentina se han llevado a cabo estudios de accesibilidad hacia servicios colectivos y de salud, para analizar desigualdades espaciales y distribución territorial como se observa en los estudios de accesibilidad geográfica al sistema de salud y educación de (Villanueba, 2010) y (Martínez Bascuñán y Rojas Quezada, 2014), unidades de servicio en general de una ciudad junto con fundamentos diseño y aplicación de (Garrocho y Campos, 2006). 
Por otro lado en países como España, Canadá, Estados Unidos y Nueva Zelanda la accesibilidad se evalúa a través de las variables contextuales/ambientales que tienen los alumnos para decidir emplear desplazamientos activos y determinar su área de influencia (Chica Olmo, Rodriguez Lopez y Chillón, 2018). Estos análisis van a acompañados de los efectos socio demográficos y psicológicos que implica elegir un modo de desplazamiento saludable (Larsen et al., 2009) (Mandic et al., 2015).

Gracias a este tipo de investigaciones, se han realizado programas de accesibilidad al espacio educativo fomentando la seguridad y la actividad física. Este planteamiento obliga a la unión de esfuerzos por parte del gobierno y los centros educativos a generar mejor infraestructura para las poblaciones y mejores condiciones de movilidad (Rossiter, 2013).

\section{Material y método}

El área de interés para el estudio es la ciudad de Ibagué también conocida como la "capital musical de Colombia". Es la capital del departamento del Tolima, localizado sobre la cordillera central de los Andes a $1285 \mathrm{msnm}$ en el centro oriente del país, a 207 km de Bogotá. Es una ciudad intermedia con 533361 habitantes en la zona urbana hacia el 2016. Actualmente, su división política administrativa contempla 13 comunas (Figura 1). Su tasa de crecimiento demográfica más alta tuvo lugar entre los años de 1951 y 1964 por el fenómeno de la violencia que forzaba el desplazamiento de la población de las zonas rurales hacia las ciudades (UN, CEPAL, CELADE, 2003). El 25\% de la población total de Ibagué, se encuentra en edad escolar con edades entre los 5 y 14 años (Departamento Administrativo Nacional de Estadística, s.f.).

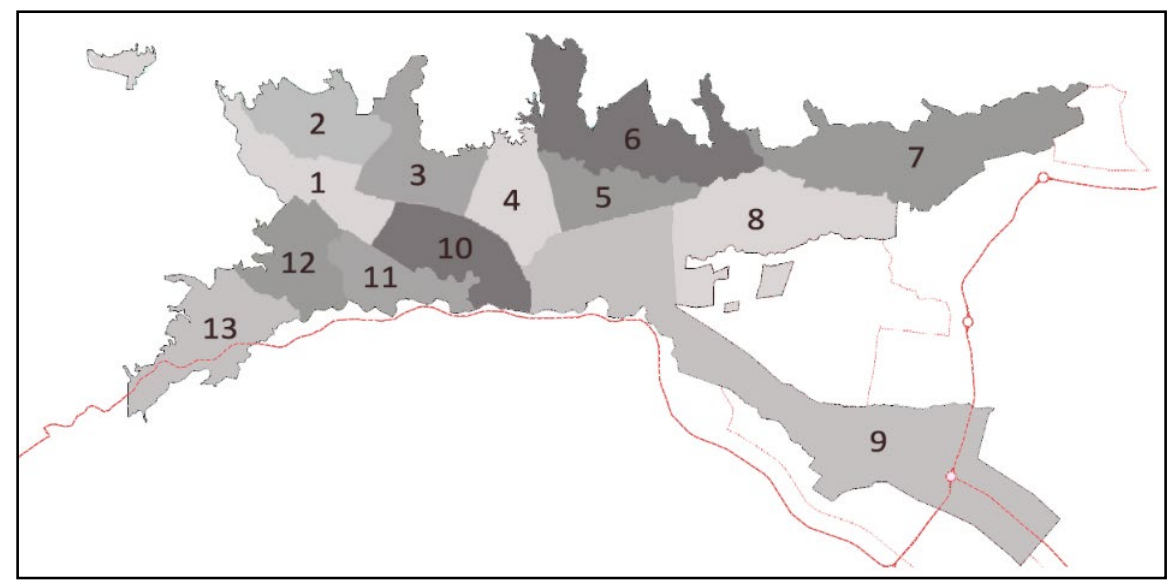

Figura 1. División político administrativa de Ibagué (Comunas). Elaboración propia en base a la Cartografía del Acuerdo 0823 de 2014 (Plan de ordenamiento territorial). 
En el entorno educativo, se identifica que los niveles de básica primaria y secundaria poseen la mayor participación sobre el territorio con un 75\% (Alcaldía de Ibagué, 2015) y coincide con el interés de este estudio en examinar la disponibilidad y accesibilidad a establecimientos que dicten dichos niveles. Este estudio posee una muestra de 201 centros educativos entre oficiales y privados sobre la zona urbana de Ibagué; en la Figura 2 se muestra la distribución espacial de los mismos.

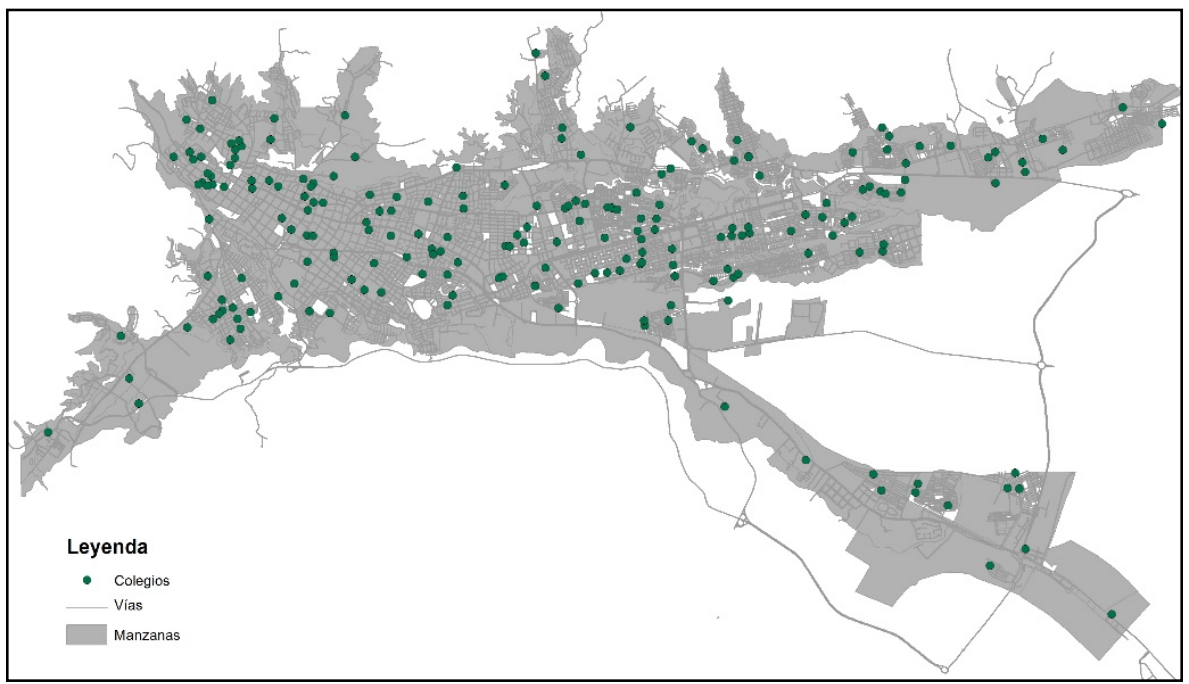

Figura 2. Ubicación espacial de centros con educación básica primaria y secundaria. Elaboración propia a partir de listado Oficial de instituciones educativa de la Gobernación del Tolima 2017 y cartografía obtenida en el Geoportal del DANE.

\section{Recolección de datos}

En principio, se obtuvo el plano vial del casco urbano de Ibagué por medio de la plataforma Open Street Map, a partir de este se filtró la información que contenía vías y senderos peatonales (Fundación Open Street Map, 2017). Dicho plano se apoyó en la cartografía de la ciudad estructurada por manzanas catastrales y obtenidas en el Geoportal DANE por código identificador de cada manzana y su área por medio del sistema de referencia MAGNA-SIRGAS "WGS 1984 Web Mercator Auxiliary Sphere".

La información demográfica por manzanas se consultó en el "Sistema de Consulta de Información Censal", que por medio de categorías filtra toda la información del único censo completo en Colombia que data del año 2005 (Departamento Administrativo Nacional de Estadística, 2007). Posteriormente, se identificó la información de grupos quinquenales por manzanas y formato de salida en tabla. De esta 
manera, la información se unió a la cartografía obtenida por medio del código de manzana y la información catastral en común.

Por otra parte, se obtuvo el listado oficial de instituciones educativas con las matrÍculas registradas a 2017 a través de la Gobernación del Tolima. Este listado identifica los grupos quinquenales por niveles de educación preescolar, básica y media. En este estudio se tuvieron en cuenta las plazas de los niveles de básica primaria y secundaria con población entre los 5 y 14 años. Adicionalmente y por medio de "Google Earth", "Google Street View" y el listado de colegios obtenidos, se reubicaron y georreferenciaron los centros educativos sobre la ciudad.

Posteriormente el estudio es divido en dos etapas: la primera contemplando los centros educativos en términos generales, es decir, públicos y privados; y la segunda hacia los centros educativos oficiales, es decir de administración pública, que corresponden a la enseñanza gratuita y obligatoria de la educación básica. Para generar la unión entre la ubicación espacial y las plazas de cada centro educativo, se generó un identificador según la categoría del establecimiento de la siguiente manera:

- 1000 - Centros

- 1100 - Colegios

- 1200 - Gimnasios y Gimnasios infantiles

- 1300 - Instituciones educativas

- 1400 - Jardines Infantiles

- 1500 - Liceos

De esta manera la unión contiene 201 centros educativos con: el código identificador, el nombre del establecimiento, el número de plazas totales, en número de plazas por educación básica primaria y secundaria y las coordenadas georreferenciadas X y Y.

En la segunda etapa del análisis solo se tienen en cuenta los centros educativos oficiales, por tanto se filtra el listado de la primera etapa solo con el identificador "1300" que corresponde al nombre de "Instituciones Educativas" y son responsabilidad de la administración pública de la ciudad. En esta etapa se obtiene un listado de 48 centros.

\section{Procesamiento de datos}

Las variables empleadas para el análisis de accesibilidad peatonal se calcularon con el apoyo ArcGIS. El proceso requiere implantar los datos obtenidos (Cartografía, demografía y educación) para la modelización del sistema urbano por medio del sistema de referencia MAGNA-SIRGAS "WGS 1984 Web Mercator Auxiliary Sphere".

En primer lugar, se determinó la densidad poblacional general y se realizó un contraste con la densidad de la población escolar (5-14 años) de forma espacial y 
cuantitativa presentada en planimetría vectorial por medio de la división del número de habitantes de cada manzana sobre su área. La densidad poblacional permitió analizar el acceso a los centros educativos a partir de la concentración de población en los diferentes puntos de la ciudad. Los campos incluidos dentro de esta planimetría contienen: el identificador de manzana, el número de habitantes por edades y la densidad poblacional expresada en hab $/ \mathrm{m}^{2}$.

En segundo lugar se asignó la impedancia sobre la red vial. El término impedancia hace referencia "a la resistencia al movimiento en un intervalo determinado y es el resultado de la longitud de los tramos sobre la velocidad del recorrido" (Loyola Gómez y Albornoz del Valle, 2009). En este análisis de accesibilidad el valor medio contemplado para la velocidad peatonal es de $4,5 \mathrm{~km}$, soportado en el estudio de (Munoz-Raskin, 2010). Posteriormente, se realizó el cálculo de la topología sobre la red creando nodos que verificaron la unión de la misma.

Por último, se definieron centroides por manzanas, es decir la población en la manzana como elemento puntual al igual que la representación de los centros educativos. De esta manera, se realizó un cálculo sobre la red por medio de la herramienta "Closet Facility" en la cual se muestran las rutas óptimas desde cada centro de manzana a cada uno de los 201 centros educativos en la primera etapa y los 48 centros oficiales en la segunda. Junto con ella se generó la matriz Origen Destino (OD), tomando como origen los centros de manzana y destino los centros educativos, de esta manera se obtiene la información correspondiente al tiempo de recorrido y distancia por medio de la red vial.

\section{Aplicación de indicadores de accesibilidad}

Una vez extraídos los tiempos de viaje, se seleccionan los indicadores que relacionen los factores físicos y sociales para analizar la accesibilidad peatonal hacia los centros educativos. Para determinar el uso correcto de los mismos, es importante tener claro el campo, la escala de aplicación, el tipo de desplazamiento, etc. (Monzon de Caceres, 1988). La distancia es el factor común entre los indicadores de origen-destino y la impedancia, puesto que la movilidad no es ilimitada (Martínez Sánchez-Mateos, 2012). Para este estudio se analizaron los tiempos mínimos de acceso, la accesibilidad absoluta, el indicador de oportunidades acumuladas en el análisis y la demanda potencial a cada centro educativo.

Debido a las implicaciones que requiere analizar la accesibilidad se han desarrollado múltiples indicadores para conseguirlo. Es importante tener claro el campo y la escala de aplicación, para seleccionar el indicador correcto; En el estudio realizado por (Monzon de Caceres, 1988) se incluye una clasificación comprensible de los indicadores según los criterios anteriores, como se muestra en la Tabla 1. 
Tabla 1

Clasificación de Indicadores de Accesibilidad

\begin{tabular}{ll}
\hline Topológicas & $\begin{array}{l}\text { Tipología del tramo de la red } \\
\text { Medidas de Trazado } \\
\text { Medidas de oposición al desplazamiento }\end{array}$ \\
De viaje & Utilización de Itinerario \\
& Probabilidad de producirse \\
Agregadas & Gravitacional \\
& Máximo beneficio del usuario \\
Desagregadas & Oportunidades acumuladas \\
& Tiempo-Espacio geográfico \\
Basadas en Transporte Público & Zona urbana \\
& Zona rural \\
\hline
\end{tabular}

Fuente: elaboración propia en base a Monzon de Caceres, 1988.

\section{Medidas desagregadas}

Este grupo de indicadores consideran el desplazamiento de un elemento individual, ya sean personas o vehículos por la red vial y no tienen presentes el resto de los elementos. Se utilizan para analizar el número de destinos que puede alcanzar un determinado usuario desde orígenes concretos (Monzon de Caceres, 1988).

\section{Indicador de Tiempos Mínimos}

Es el cálculo del tiempo mínimo desde cualquier centro de manzana como origen hacia el centro educativo más cercano. Para esto se tuvo en cuenta, además de la distancia, la red vial y la velocidad media del peatón andando: $4.5 \mathrm{~km} / \mathrm{h}$ (MunozRaskin, 2010).

\section{Indicador de Accesibilidad Absoluta}

Este indicador es sensible a la localización geográfica de los nodos, mide el grado de interconexión de un punto con el resto de puntos de la región estudiada basándose en el coste del transporte y en los modelos potenciales de mercado primando siempre las zonas centrales. Para este caso se calculó el promedio de las impedancias que separan a cada elemento puntual que concentra la población en las manzanas respecto a los centros educativos a través de la red, además se considera la capacidad de cada centro como factor de ponderación a partir de la Ecuación (1).

$$
I A A=\frac{\sum_{j=1}^{n}\left(I_{I j} * C C_{j}\right)}{\sum_{j=1}^{n} C C_{j}}
$$


Donde:

Iij: es el tiempo mínimo entre los nodos ij, a través de la red, es decir, la impedancia entre origen y destino.

$C C j$ : es la capacidad educativa de cada centro sobre la zona urbana.

El respaldo gráfico de este indicador es un mapa vectorial que en conjunto con la información tabular representan por tonalidades el nivel de accesibilidad que se posee hacia la zona de mayor actividad.

\section{Indicador de Oportunidades Acumuladas}

En este indicador se contabilizó la cantidad de población escolar entre los 5 y 14 años que está a 15 minutos de distancia, con respecto a uno o varios centros educativos, definiendo la demanda potencial existente en su entorno; esta se contrasta posteriormente con la capacidad de plazas que posea cada colegio.

\section{Resultados y discusión}

Para generar un diagnóstico global de la accesibilidad al servicio educativo hacia los centros, se requirió de una visión espacial y cuantitativa de los resultados que tiene en cuenta la localización e implicaciones de desplazamiento junto con la oferta y demanda de cada uno. El método de estudio por indicadores en este análisis, amplió la idea de que la accesibilidad está ligada únicamente con la ubicación de los centros, definiendo la cobertura educativa y la capacidad de atención por sectores.

\section{Densidad poblacional general y escolar}

Este indicador permitió observar la configuración espacial de la ciudad, en primera instancia con la distribución de la población sobre el territorio y en segunda la población en edad escolar entre 5 y 14 años que reciben la educación básica primaria y secundaria (Figura 3); su unidad de medida se representa en hab $/ \mathrm{km}^{2}$.

A nivel espacial, ambas densidades se ajustan en distribución geográfica sobre el territorio, lo que permite identificar integración de la población sin discriminación por edades.

A nivel cuantitativo por medio de la Figura 4, se puede analizar que la densidad escolar media es de $6448 \mathrm{hab} / \mathrm{Km}^{2}$ y representa el $20 \%$ de la densidad poblacional general. También se muestra que el $18 \%$ de las manzanas que posee una densidad baja entre los 10 a $2500 \mathrm{hab} / \mathrm{km}^{2}$, seguido de un $63 \%$ que concentra una densidad media entre 2500 a $9500 \mathrm{hab} / \mathrm{km}^{2}$. Finalmente un $18 \%$ con una densidad alta superior a dicha cifra. 


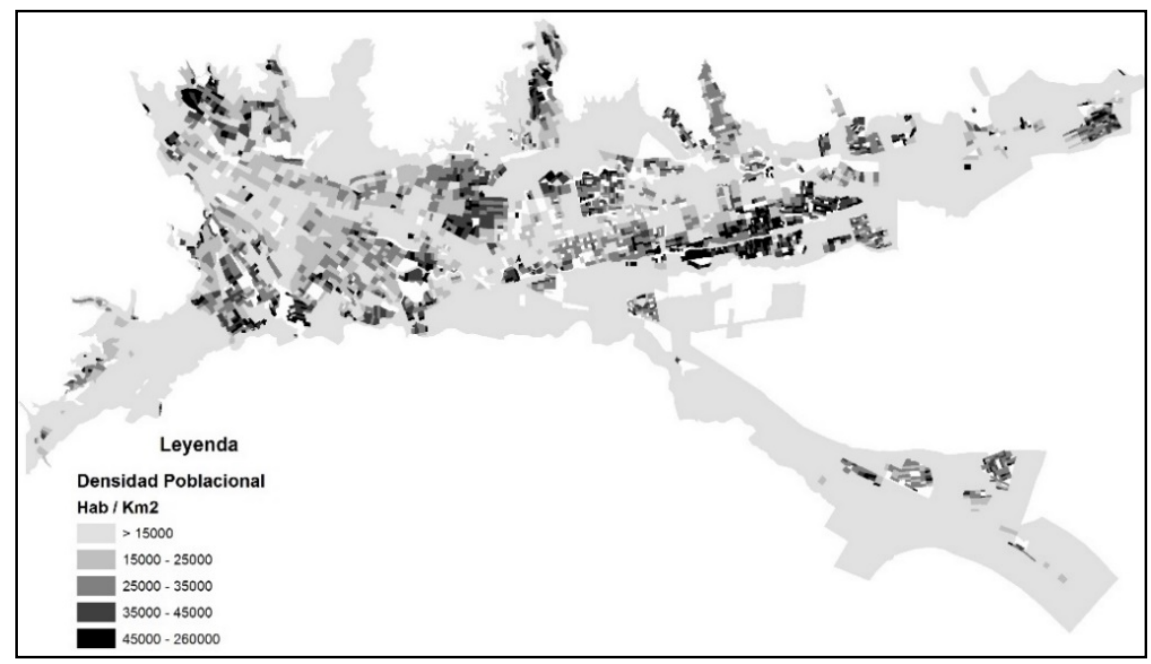

Figura 3. Densidad de la población entre los 5 y 14 años de Ibagué.

Elaboración propia en base al censo poblacional 2005, DANE.

\section{Densidad poblacional escolar hab $/ \mathrm{km}^{2}$}

10 a 2.500

2.500 a 4.500

4.500 a 6.500

- 6.500 a 9.500

- 9.500 a 42.500

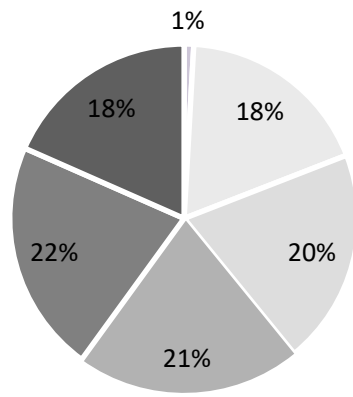

Figura 4. Diagrama por sectores de la densidad poblacional escolar. Fuente: elaboración propia a partir de DANE, 2005.

\section{Indicadores: Centros educativos generales}

\section{Indicador de tiempos mínimos}

En la Figura 5 se muestra el mapa de tiempos mínimos de acceso peatonal desde cualquier manzana al centro educativo más cercano. A través de este indicador se 
observa el grado de accesibilidad que posee la población por sectores a los centros educativos más cercanos.

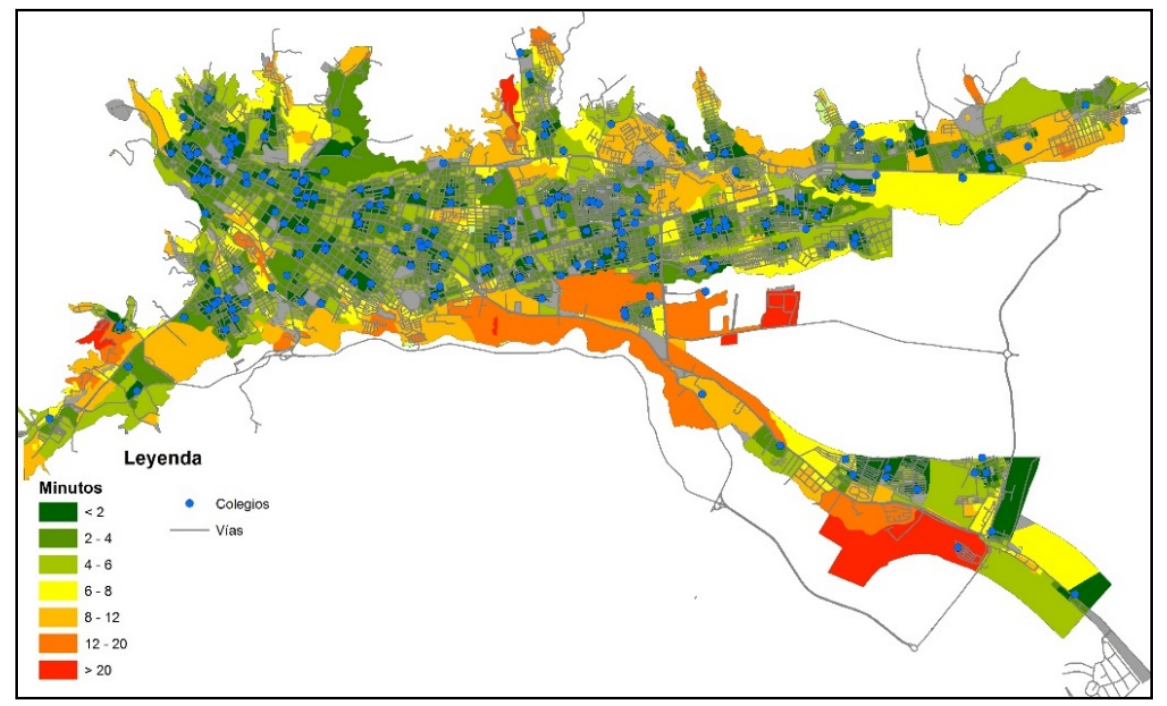

Figura 5. Indicador de Tiempos Mínimos de acceso peatonal hacia los Centros Educativos de Ibagué.

Espacialmente, se observa que gran parte de la ciudad posee una accesibilidad alta a menos de seis minutos hacia los centros educativos, representada en tonos verdes. Este patrón se presenta sobre el cuerpo central de la ciudad y se pierde sobre la periferia en las comunas 7, 9 y 13 cuando la accesibilidad corresponde a un desplazamiento entre 6 a 20 minutos andando, representado en tonos amarillos y naranjas. Por otra parte, existen zonas puntuales sin configuración previa que tienen una accesibilidad mayor a 20 minutos representadas en color rojo, estas se ubican sobre la periferia y su presencia es mínima respecto al resto del territorio. Las zonas con un tono gris, corresponden a aquellas que no tienen información poblacional, por ser espacio público o equipamientos colectivos.

Cuantitativamente, se confronta la distribución del porcentaje de población por medio de la Figura 6 y se obtiene que más del $80 \%$ de la misma tiene un colegio a menos de seis minutos de distancia, situándose geográficamente sobre el cuerpo principal de la ciudad y algunas zonas de la periferia como se observa en la Figura 4. En algunos sectores, la accesibilidad supera los seis minutos y se debe a la falta vías alternas de comunicación. Solo un porcentaje inferior al $1 \%$ posee una accesibilidad baja mayor a 20 minutos andando, estas hacen referencia a zonas que están en proceso de consolidación o en las que carecen de vías comunicación como en las comunas 7 , 9 y 13. 
Tiempos mínimos de acceso en minutos

$$
\begin{aligned}
& =66 \mathrm{~min} \\
& 6-12 \mathrm{~min} \\
& 12-20 \mathrm{~min} \\
& =20 \mathrm{~min}
\end{aligned}
$$

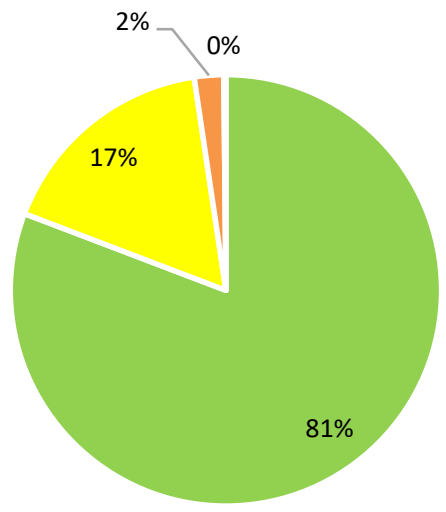

Figura 6. Diagrama por sectores del grado de accesibilidad en el indicador de Tiempos Mínimos.

\section{Indicador de accesibilidad absoluta}

Este indicador muestra el grado de accesibilidad peatonal según la localización de la población al conjunto de centros educativos. A partir de la zona central se generan cinco anillos concéntricos que reducen el grado de accesibilidad conforme llegan a las periferias. De esta manera, el indicador cumple el principio de "centro-periferia" mostrando que la población localizada en el centro de la ciudad, tiene mayor posibilidad de acceso al conjunto de centros educativo que los ubicados en alguna isla periférica.

A nivel espacial, se observa que dicho principio corresponde con una alta accesibilidad en el centro de la ciudad y es representada por tonos verdes sobre las comunas 4 y 5 junto con partes de las comunas 6 y 10; a medida que se extiende, el grado de accesibilidad disminuye concentrándose en rojo como se observa sobre las comunas 7, 9 y 13 en la Figura 7. La característica particular de estas zonas es la configuración territorial respecto al cuerpo principal de la ciudad lo que genera reducción significativa de vías de comunicación. Desde un enfoque cuantitativo, se observa en la Figura 8 una media aproximada del 33.31\% sobre la distribución de accesibilidad Alta, Media y Baja confirmando el principio de centro periferia. Sin embargo, la baja accesibilidad posee un aumento del $2 \%$ por encima de dicha media que afecta a las comunas periféricas 7,9 y 13 . 


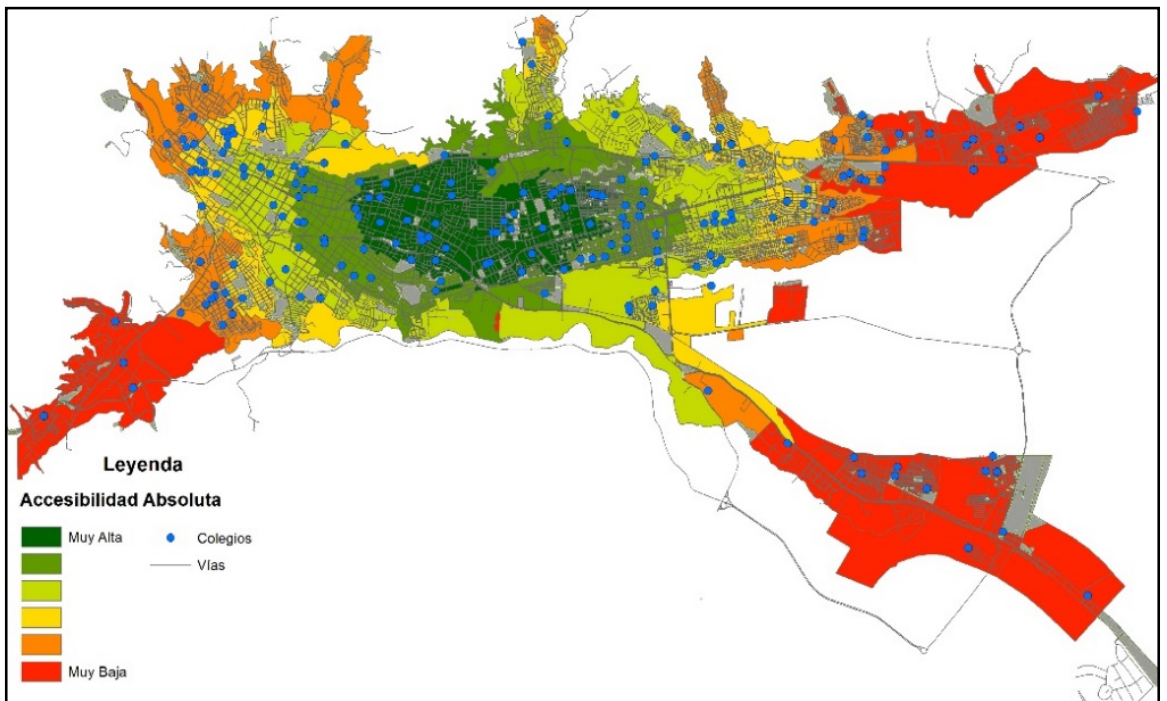

Figura 7. Indicador de Accesibilidad Absoluta peatonal hacia los Centros Educativos de Ibagué.

\section{Accesibilidad absoluta por manzanas}

$$
\begin{aligned}
& -<62 \mathrm{~min} \\
& -62-77 \mathrm{~min} \\
& ->77 \mathrm{~min}
\end{aligned}
$$

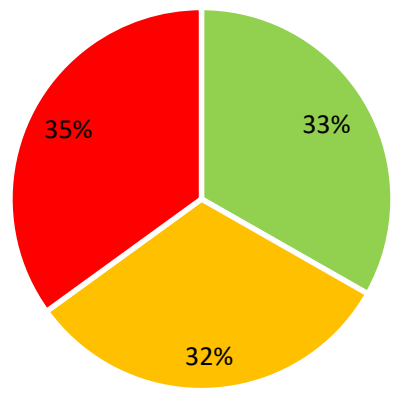

Figura 8. Diagrama por sectores del indicador de Accesibilidad Absoluta por minutos.

Demanda potencial a cada centro educativo

En este cálculo, se define para cada centro educativo la demanda de población escolar entre los 5 y 14 años más cercana por manzanas. Este indicador permite realizar una 
comparación con la oferta de cada centro y evaluar la capacidad de atención hacia la población inmediata.

En la Figura 9 se muestra la localización de los centros educativos a partir de la demanda cercana a cada uno por sector. Además, se observa que los centros con una población cercana menor a los 800 habitantes se distribuyen linealmente sobre el centro de la ciudad y los superiores a esta cifra se localizan sobre las zonas más densas correspondientes a sectores de las comunas 2, 6, 7, 8 y 12 .

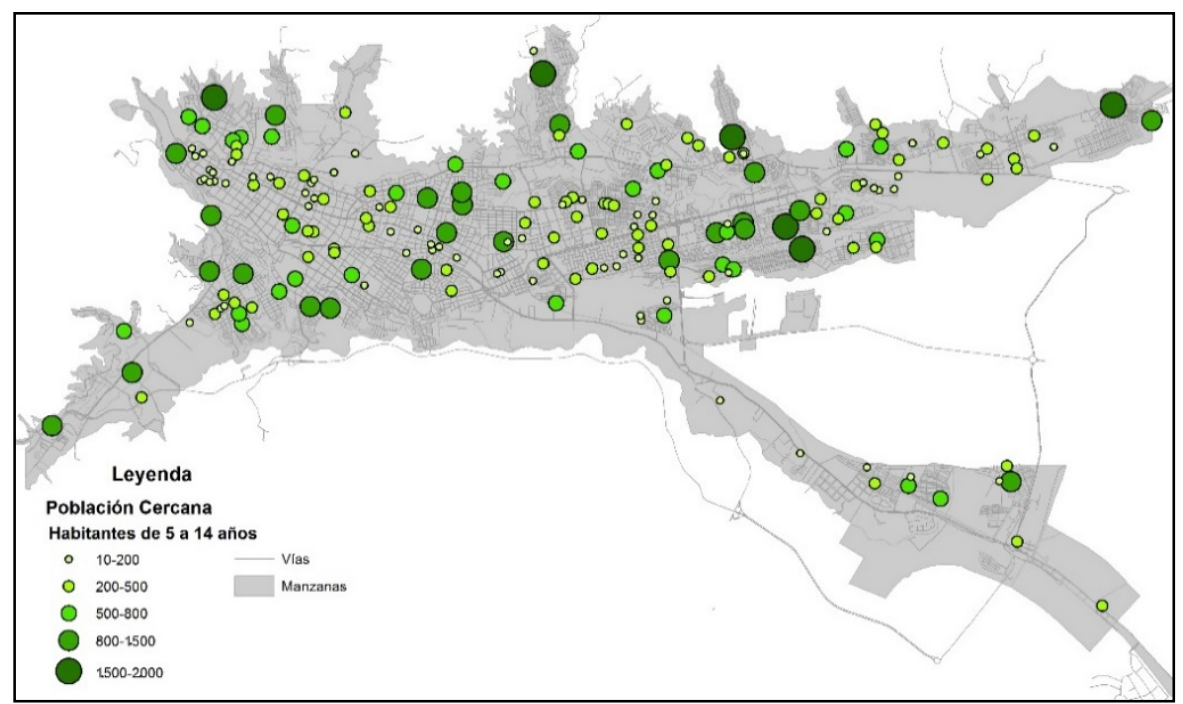

Figura 9. Población Potencial Cercana a cada Centro educativo.

De forma cuantitativa a través de la Figura 10, se encontró que el $80 \%$ de los centros tiene una población cercana menor de 800 habitantes y se localizan sobre el cuerpo principal de la ciudad, seguido del $20 \%$ que posee una demanda superior a dicha cifra y su localización es dispersa. También, se obtiene que la media de la población cercana es de 426 habitantes, y se distribuye de forma constante sobre el diagrama de líneas. Adicionalmente, se confirma que, de los 201 centros educativos, solo el $29.95 \%$ suple la demanda existente en su sector, el resto de centros posee tasas de ocupación entre el $150 \%$ y el $920 \%$, superando la oferta de la mayoría de centros.

\section{Indicadores de oportunidades acumuladas a 15 minutos}

Por medio de este indicador se determina la posibilidad que tiene la población escolar en acceder peatonalmente a cualquier centro educativo a menos de 15 minutos.

Espacialmente por medio de la Figura 11, se muestra que la población del cuerpo central de la ciudad tiene más posibilidad de acceso a varios centros educativos con 
templando cifras entre los 1500 a 9000 habitantes. Los centros con mayor demanda entre los 7000 y 9000 habitantes, corresponden con la densidad poblacional escolar expuesta en la Figura 4. Por otra parte, se observa que las comunas 9 y 13 presentan demandas inferiores a 1500 habitantes por centro educativo.

Demanda y oferta por centro educativo

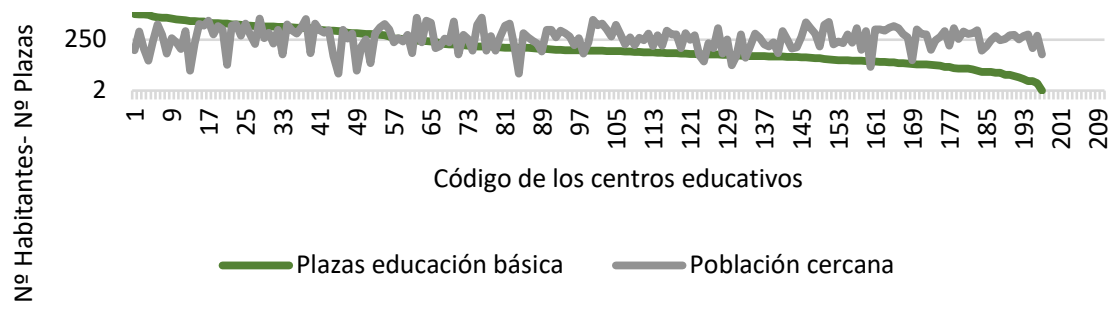

Figura 10. Diagrama de líneas que contrasta la oferta de cada centro educativo respecto a la demanda cercana por sector.

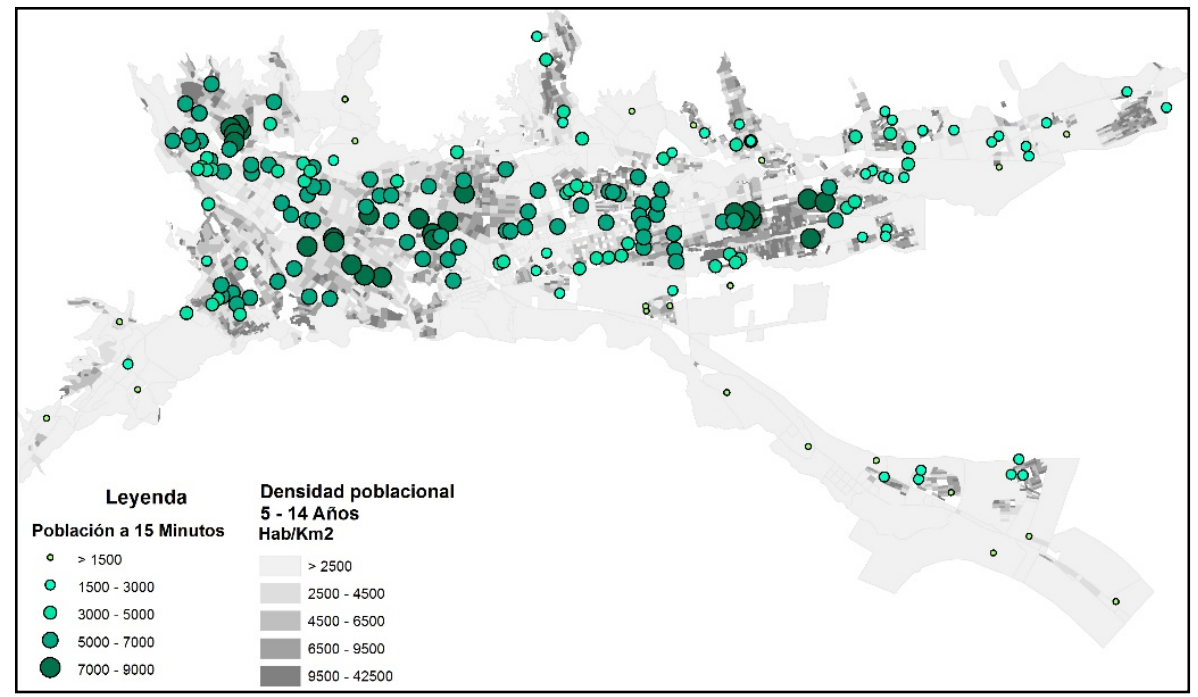

Figura 11. Indicador de Oportunidades acumuladas: Población potencial a menos de 15 minutos de los centros educativos en contraste con la densidad poblacional escolar.

Cuantitativamente se analiza por medio de la Figura 12, que la media de población demandante es de 4368 habitantes en confrontación con la media de plazas ofertadas en los centros que son 368 superando casi en 11 veces su capacidad. Adicionalmente, 
se obtuvo que el $46 \%$ de los centros educativos poseen una demanda cercana mayor a 5000 habitantes a menos de 15 minutos de distancia, seguido de un $43 \%$ entre 1500 y 5000 habitantes, estos dos porcentajes se distribuyen sobre el cuerpo central de la ciudad. Finalmente, un $10.14 \%$ de los centros con población cercana menor a 1500 habitantes que se ubica sobre la periferia. Respecto a la respuesta a esta demanda, solo el $4.3 \%$ de los centros tiene capacidad de atención, lo que implica desplazamiento de la población.

Demanda a 15 minutos de centros educativos

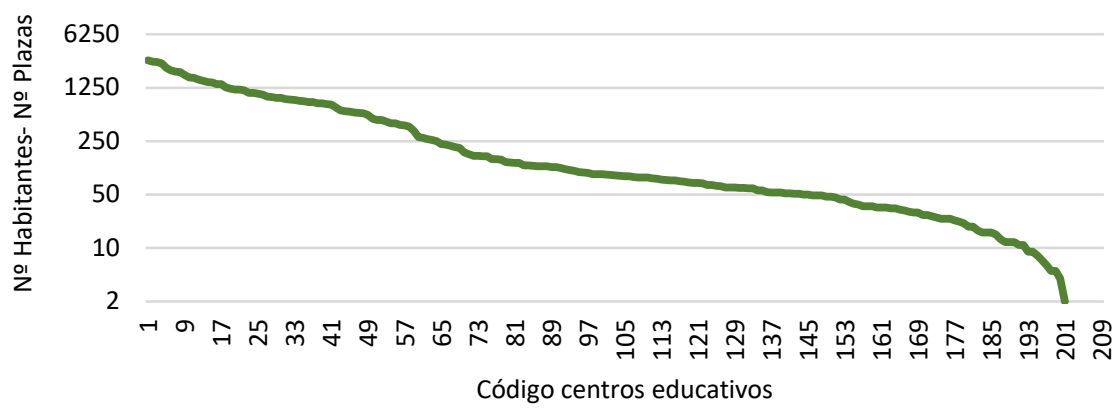

Población a 15 minutos Plazas educación básica

Figura 12. Diagrama de líneas que contrasta la oferta de cada centro educativo y la demanda potencial a 15 minutos de distancia.

\section{Indicadores: Centros educativos oficiales}

El desarrollo de esta etapa permite evaluar el estado de la accesibilidad a los centros educativos de carácter oficial que son administrados por el estado y deben brindar un servicio equilibrado y accesible a la población en edad escolar, por tanto tener la capacidad suficiente de atención a la demanda por sectores.

\section{Distribución espacial de centros educativos oficiales}

Por medio de la Figura 13 se muestra la distribución espacial de los 48 centros educativos de carácter oficial en la ciudad de Ibagué. Esta distribución, añade que existe baja presencia de centros sobre las comunas 1 y 4, ubicadas en el cuerpo central de la ciudad y sobre las comunas 7, 9 y 13 ubicadas en la periferia.

\section{Indicador de tiempos mínimos}

A nivel espacial y por medio de la Figura 14 los sectores con una accesibilidad alta inferior a los 10 minutos de un centro educativo se encuentran fragmentados en dos sobre la comuna 4 en el centro de la ciudad con accesibilidad reducida a un tiempo 
entre los 10 a 15 minutos. Por otra parte, las comunas 3, 6, 7, 9 y 13 se afectan parcialmente entre los 15 y 20 minutos. Finalmente, sobre la comuna 9 se muestra como la accesibilidad baja es la más pronunciada con tiempos superiores a los 20 minutos.



Figura 13. Ubicación espacial de Centros con Educación Oficiales en la ciudad de Ibagué. Fuente: Elaboración propia a partir de listado Oficial de instituciones educativa de la Gobernación del Tolima 2017 y cartografía obtenida en el Geo portal del DANE.

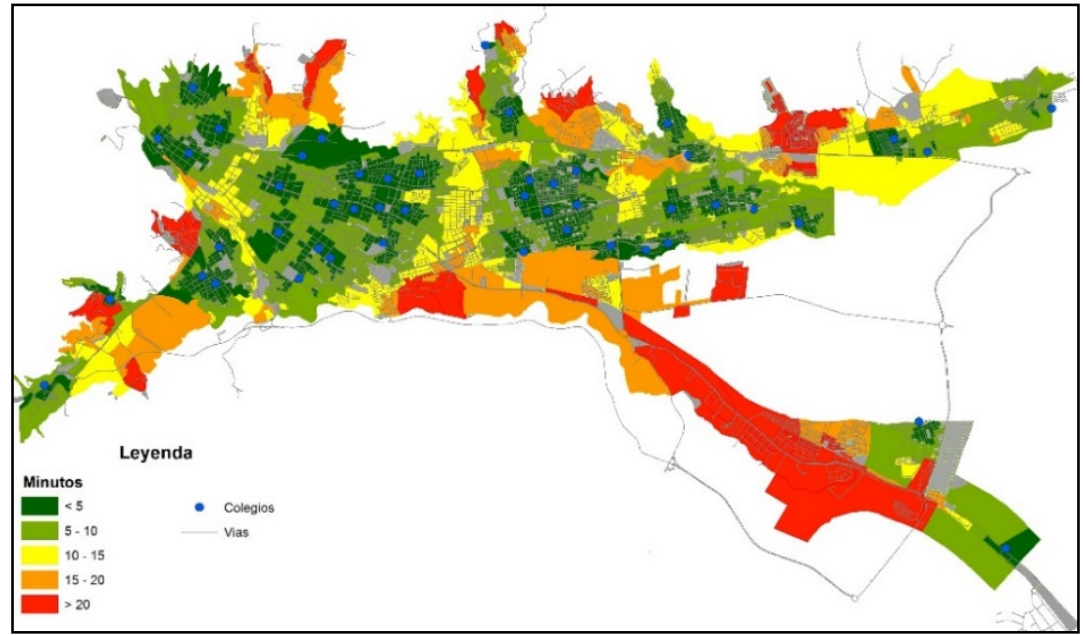

Figura 14. Indicador de Tiempos Mínimos de acceso peatonal hacia los Centros Educativos Oficiales de Ibagué. 
En términos cuantitativos, por medio de la Figura 15 se observa que el $71 \%$ de la población posee un centro educativo oficial a menos de 10 minutos de distancia. Seguido de un $23 \%$ que comprende tiempos entre los 10 a 20 minutos andando; finalmente, se muestra un 6\% de accesibilidad baja que supera los 20 minutos de desplazamiento peatonal.

Tiempos mínimos de acceso en minutos

$=<10$
$=10$ a 15
$=15$ a 20
$->20$



Figura 15. Diagrama por sectores del grado de accesibilidad en el indicador de Tiempos mínimos a los Centros Educativos Oficiales.

\section{Indicador de accesibilidad absoluta}

A través de la Figura 16 se observa el principio de centro-periferia característico de este indicador. Al mismo tiempo, se muestra que la accesibilidad alta es representada en tonos verdes y contempla distancias inferiores a los 51 minutos andando. Adicionalmente, se observa una accesibilidad baja representada en tonos cálidos sobre las comunas 7, 9 y 13 que indica tiempos superiores a 85 minutos. Adicionalmente, se demarcan dos tipos de secciones: las primeras en tono gris, dispersas irregularmente sobre la ciudad que representan zonas sin información demográfica que corresponden a espacio público o sectores sin desarrollo urbanístico. La segunda en rojo, que muestran zonas con dificultad de accesibilidad por ausencia de vías de comunicación.

Dentro del análisis cuantitativo, se obtiene que el $40 \%$ de la población posee una accesibilidad buena hacia el conjunto de centros educativos con tiempos inferiores a los 65 minutos. Seguido de una accesibilidad media representada en otro $40 \%$ y finalmente, una accesibilidad baja con un $20 \%$ confirmando lo observado en la Figura 17. 


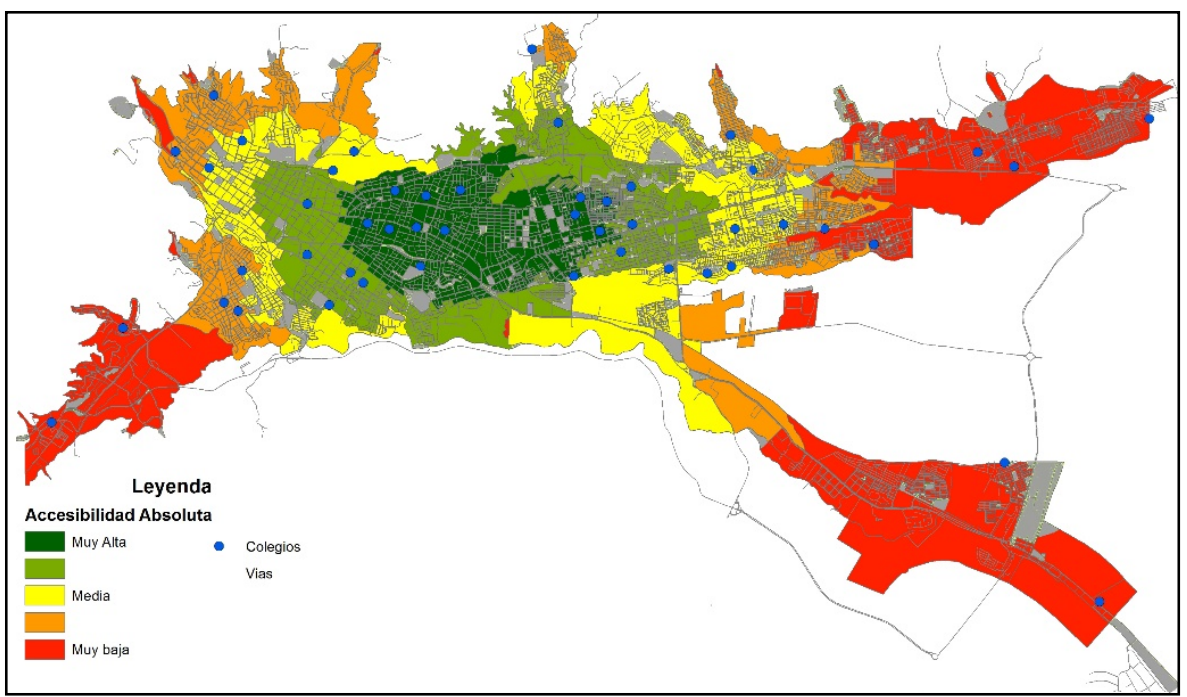

Figura 16. Indicador de Accesibilidad Absoluta peatonal hacia los centros educativos oficiales de Ibagué.

Accesibilidad absoluta por manzanas en minutos

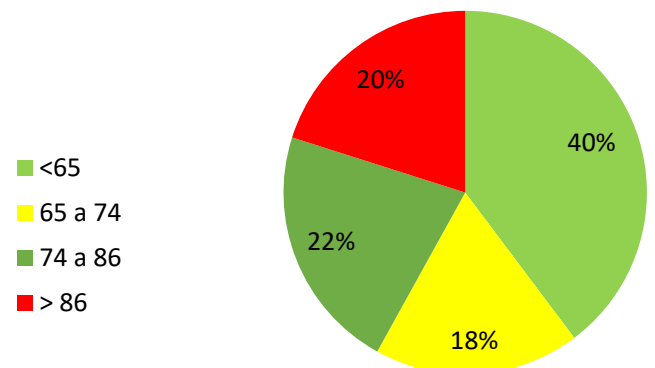

Figura 17. Diagrama por sectores del indicador de Accesibilidad Absoluta por minutos al conjunto de Centros Educativos Oficiales.

Demanda potencial a cada centro educativo oficial

Espacialmente, como se muestra en la Figura 18 la demanda potencial hacia la mayoría de centros educativos oficiales corresponde con zonas de alta densidad poblacional superando a los 2300 habitantes por centro. 


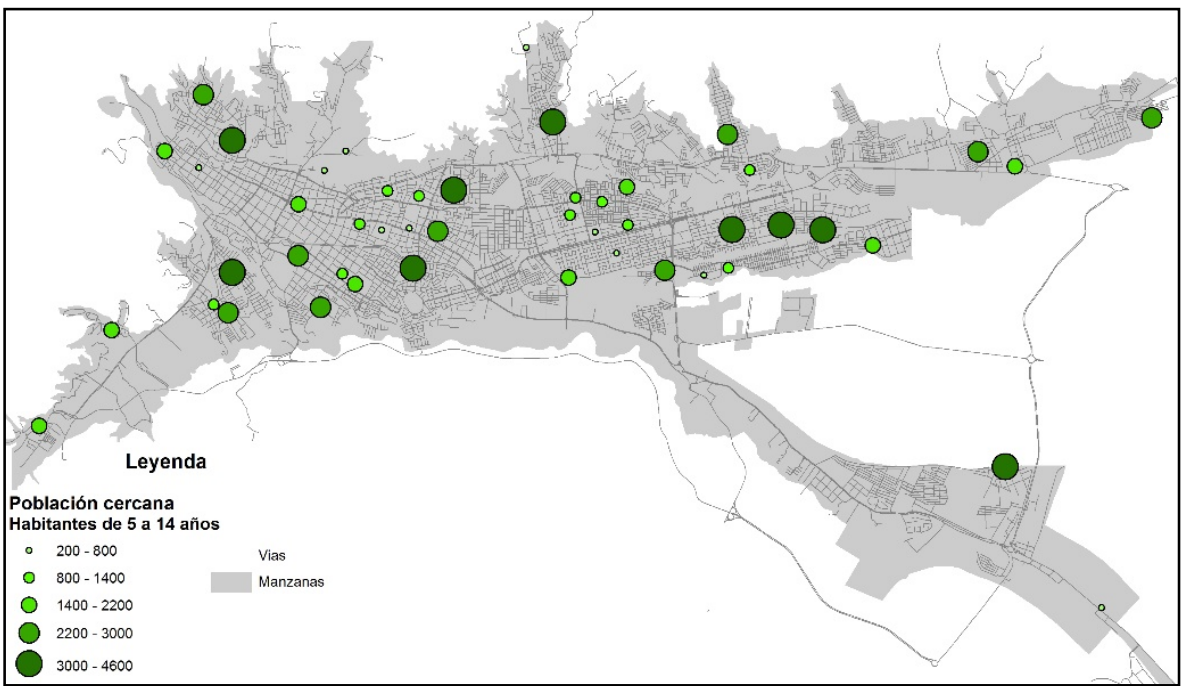

Figura 18. Población Potencial Cercana a cada Centro Educativo Oficial.

Cuantitativamente, se muestra en la Figura 19 que el $39.58 \%$ de los centros posee una demanda potencial menor a los 1500 habitantes, seguido de un $22.65 \%$ con una demanda entre los 1500 y 2300 habitantes, finalmente se muestra que el $37.5 \%$ supera dicha cifra. En cuanto a la atención de la demanda por sectores, solo el 13\% es apto para soportarla.

Oferta y demanda por centro educativo oficial

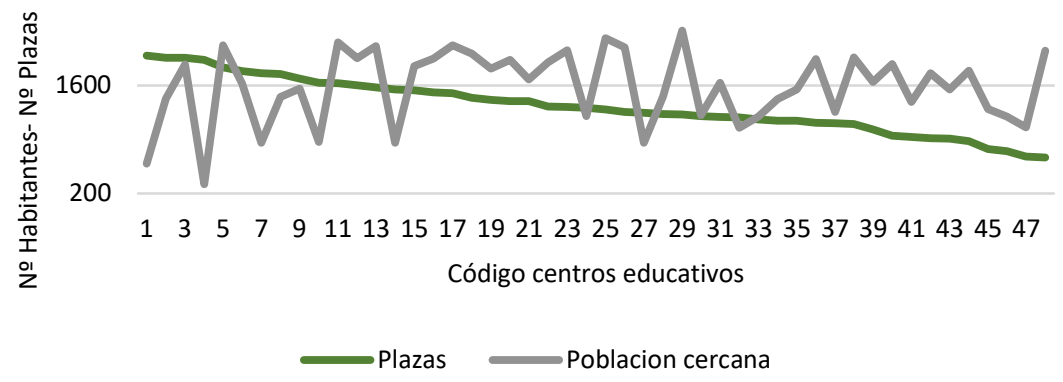

Figura 19. Diagrama de líneas que contrasta la oferta de cada centro educativo oficial respecto a la demanda cercana por sector. 


\section{Indicador de oportunidades acumuladas a 15 minutos}

Espacialmente, la concentración de la demanda se sitúa sobre las comunas 3, 5, 8, 10 y 11, con cifras superiores a los 5600 habitantes. Las zonas con menor concentración de demanda se localizan sobre la periferia en las comunas 7, 9 y 13 como se observa en la Figura 20.

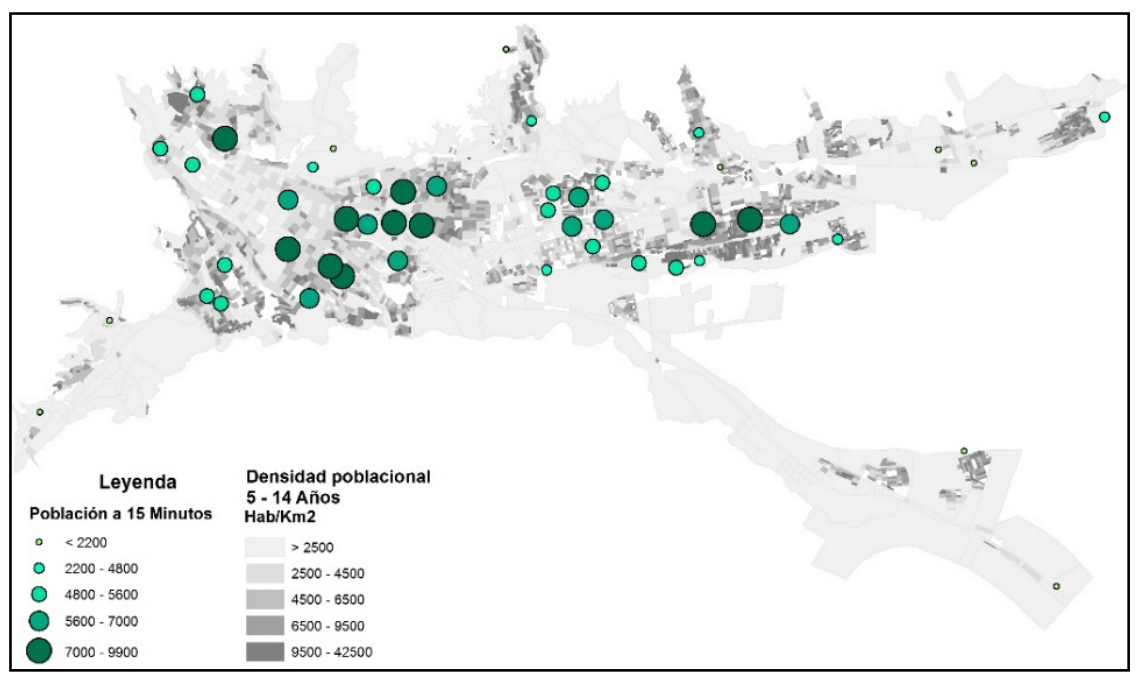

Figura 20. Mapa del indicador de Oportunidades Acumuladas: Población potencial a menos de 15 minutos de los Centros Educativos Oficiales en contraste con la densidad poblacional escolar.

Cuantitativamente y por medio de la Figura 21, el 39.58\% de los colegios posee alta demanda superior a los 5600 habitantes a 15 minutos de distancia. Seguido de un $18.75 \%$ entre los 4800 a 5600 habitantes, un $22.91 \%$ entre los 2200 ha 4800 habitantes y finalmente, un $18.75 \%$ con una demanda inferior a los 2200 habitantes. De estas cifras, el $12.5 \%$ de los centros tienen capacidad de atención para la demanda aportada en este filtro de 15 minutos.

\section{Conclusiones}

La población en edad escolar entre los 5 a 14 años tiene una accesibilidad peatonal baja hacia los centros educativos oficiales y privados que ofrecen los niveles de educación básica primaria y secundaria en la ciudad de Ibagué. Los resultados muestran que existe un déficit de atención en un servicio de carácter obligatorio que el estado y las entidades internacionales vigilan y hace parte del cumplimiento de los derechos humanos como es la educación. 
Oferta y demanda a 15 minutos de centros educativos oficiales

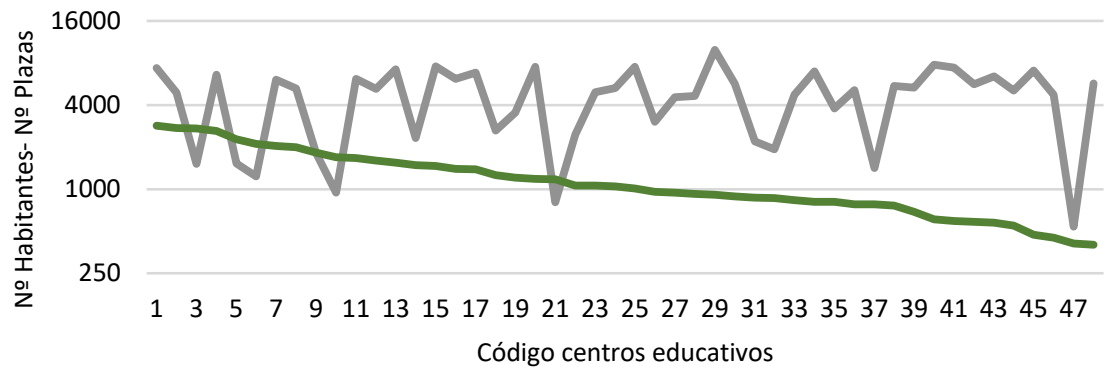

$\longrightarrow$ Poblacion a 15 Min $\longrightarrow$ Plazas

Figura 21. Diagrama de líneas con Demanda Potencial a 15 minutos de distancia hacia los Centros Educativos Oficiales.

En cuanto a la localización de los centros educativos, a nivel general corresponde en un $80 \%$ a la densidad poblacional escolar de la ciudad y a nivel oficial la cifra se reduce a un $60 \%$ aproximadamente. En la etapa de centros educativos generales, la concentración de los mismos se sitúa sobre el cuerpo principal de la ciudad, debilitando las comunas 7, 9 y 13 que corresponden a las alas periféricas. En la etapa de centros educativos oficiales, la concentración no solo afecta a dichas zonas, también se muestra poca presencia de centros sobre las comunas 1 y 4 localizadas al oriente y centro de la ciudad respectivamente. En ambas etapas, la ubicación de los centros se considera desequilibrada, puesto que no existe un patrón de concentración justificado que fortalezca la atención a la población por sectores.

Respecto al grado de accesibilidad espacial al servicio educativo tanto general como oficial, se afirma que es bueno. Hacia los centros educativos en general el $80.78 \%$ de la población escolar ibaguereña tiene un centro a menos de 6 minutos de distancia. En los centros educativos oficiales un 71\% posee una cercanía con la población a menos de 10 minutos. Ambas situaciones se muestran sobre el cuerpo principal de la ciudad, a diferencia las zonas con accesibilidad baja que se posan sobre las comunas 7, 9 y 13 ubicadas en la periferia.

En resumen, a pesar de que la accesibilidad espacial sobre el territorio es buena, la atención a la demanda escolar no lo es. Por tanto, los costes en los desplazamientos peatonales aumentan y dificultan el acceso al servicio por la necesidad de emplear más tiempo y medios motorizados para tal fin. Es decir, que el problema va más allá de la ubicación geográfica de los centros involucrando la oferta y demanda por centro educativo. 


\section{Bibliografía}

Alcaldía de Ibagué (2015). “Ibagué cóomo vamos”, Informe de calidad de vida Ibagué 2015. Disponible en

$<$ http://www.ibaguecomovamos.org/index.php/informe/informe-de-calidad-devida $>$. Recuperado el 03 de mayo de 2017.

Chica Olmo, J., Rodriguez Lopez, C. and Chillón, P. (agosto, 2018). "Effect of distance from home to school and spatial dependence between homes on mode of commuting to school", Journal of Transport Geography, no. 72, pp. 1-12. DOI: 10.1016/j.jtrangeo.2018.07.013

Departamento Administrativo Nacional de Estadística. (s.f.). $<$ https://www.dane.gov.co/index.php/estadisticas-por-tema/demografia-ypoblacion/proyecciones-de-poblacion>. Recuperado el 16 de mayo de 2017.

Departamento Administrativo Nacional de Estadística (2007). Sistema de Consulta Información Censal, Censo Básico 2005, disponiuble en: http://systema59.dane.gov.co/cgibin/RpWebEngine.exe/PortalAction?\&MODE $=$ MAIN\&BASE $=$ CG2005BASICO\&MAIN=WebServerMain.inl $>$. Recuperado el 03 de febrero de 2017.

Departamento Nacional de Estadística (17 de diciembre de 2017). Censo 2018 $D A N E$. Línea de tiempo: los censos en Colombia: https:/censo2018.dane.gov.co/index.php/linea-de-tiempo-los-censos-encolombia $>$. Recuperado el 28 de octubre de 2018.

Fundación Open Street Map. (2017). $<$ https://www.openstreetmap.org/export\#map= 13/4.4253/-75.1860>. Recuperado el 01 de febrero de 2017.

Gallego, G., Labrador, R., Cabanillas, J. y Pintor, P. (2013). Diseño de un modelo de asignación de viajes con aplicaciones SIG para la gestión de planes de movilidad urbana sostenibles en ciudades medias (13_1).

Garrocho, C. y Campos, J. (2006). Un indicador de accesibilidad a unidades de servicios clave para ciudades mexicanas: fundamentos, diseño y aplicación, $\mathrm{VI}(22)$.

Gutiérrez Gallego, J.A., Berrocal Nieto, R. y Ruiz Labrador, E.E. (2014). Análisis de la Accesibilidad al Autobus Urbano de Mérida, (64).

Larsen, K.; Gilliland, J.; Hess, P.; Tucker, T.; Irwin, J. and He, M. (marzo de 2009). "The influence of the physical enviroment and sociodemographic characteristics o children'smode of travel to and from school", Revista Americana de Salud Pública, 99(3). DOI: 10.2105 / AJPH.2008.135319

Loyola Gómez, C. y Albornoz del Valle, E. (2009). Flujo, Movilidad y niveles de accesibilidad en el centro de chillan año 2007, 12(19).

Mandic, S.; León de la Barra, S.B García Bengoechea, E.; Stevens, E.; Flaherty, C.; Moore, A. and Skidmore, P. (2015). "Personal, social and environmental 
correlates of active transport to school among adolescents in Otago, New Zealand", Journal of Science and Medicine in Sport, 18(4):432-437.

Martínez Bascuñán, M. y Rojas Quezada, C. (2014). "Evaluación de la accesibilidad espacial a la red hospitalaria en el área metropolitana de Concepción”, GeoFocus, 2(13):176-200.

Martínez Sánchez-Mateos, H.S. (2012). La accesibilidad regional y el efecto territorial de las infraestructuras de transporte. Aplicación en Castilla-La mancha, (59).

Ministerio de Educación Nacional (31 de mayo de 2010). MINEDUCACION. disponivle en $<$ http://www.mineducacion.gov.co $>$. Recuperado el 10 de mayo de 2017.

Monzon de Caceres, A. (1988). "Los indicadores de accesibilidad y su papel decisor en las inversiones en infraestructuras de transporte. Aplicaciones en la comunidad de Madrid", tesis doctoral, Universidad Politécnica de Madrid, Mdrid. Disponible en <http://oa.upm.es/736/>. Recuperado el 12 de abril de 2017.

Mora Aliseda, J., Nogales Galán, J., Gutierréz Gallego, J. y Cortés Ruíz, T. (2003). Aplicación de técnicas de SIG en la planificación del transporte en Extremadura, 38(75), España.

Munoz-Raskin, R. (2010). "Walking accessibility to bus rapid transit: Does it affect property values? The case of Bogotá, Colombia", Transport Policy, 17(2).

NU-CEPAL-CELADE (noviembre, 2003). Estudio sobre la distribución espacial de la población en Colombia, CEPAL Ed. de Comisión Econónima para América Latina y el Caribe, disponible en:

https://repositorio.cepal.org/bitstream/handle/11362/7186/1/S0311812_es.pdf $>$. Recuperado el 28 de octubre de 2018.

Rossiter, K. (2013). Safe Routes to School Plan 2013. Recuperado el 28 de octubre de 2018, de City of Perham Minesota:

https:/www.ci.perham.mn.us/vertical/sites/\%7B5BCEF176-6AD1-406C-

A4C6-7F7D132DF5EB\%7D/uploads/Perham_FINAL_PLAN.pdf

UNESCO (2011). La UNESCO y la Educación "toda persona tiene derecho a la educación", disponible en

$<$ http://unesdoc.unesco.org/images/0021/002127/212715s.pdf $>$. Recuperado el 25 de mayo de 2017.

Villanueba, A. (2010). "Accesibilidad Geográfica a los sistemas de salud y educación”. Análisis espacial de las localidades de Necochea y Quequén (2), pp.158-177. 